\title{
The lowest Landau level in QCD
}

\author{
Falk Bruckmann ${ }^{1, a}$, Gergely Endrődi ${ }^{2}$, Matteo Giordano ${ }^{3}$, Sándor D. Katz ${ }^{3}$, Tamás G. Kovács ${ }^{4}$, \\ Ferenc Pittler ${ }^{5}$, and Jacob Wellnhofer ${ }^{1}$ \\ ${ }^{1}$ Universität Regensburg, Institut für Theoretische Physik, Universitätsstraße 31, 93053 Regensburg, Ger- \\ many \\ ${ }^{2}$ Institute for Theoretical Physics, Goethe University, Max-von-Laue-Strasse 1, 60438 Frankfurt am Main, \\ Germany \\ ${ }^{3}$ Institute for Theoretical Physics, Eötvös University, and MTA-ELTE Lattice Gauge Theory Research Group, \\ Pázmány P. sétány 1/A, H-1117 Budapest, Hungary \\ ${ }^{4}$ Institute for Nuclear Research of the Hungarian Academy of Sciences, Bem tér 18/c, H-4026 Debrecen, \\ Hungary \\ ${ }^{5}$ HISKP (Theory), University of Bonn, Nussallee 14-16, D-53115, Bonn, Germany
}

\begin{abstract}
The thermodynamics of Quantum Chromodynamics (QCD) in external (electro-)magnetic fields shows some unexpected features like inverse magnetic catalysis, which have been revealed mainly through lattice studies. Many effective descriptions, on the other hand, use Landau levels or approximate the system by just the lowest Landau level (LLL). Analyzing lattice configurations we ask whether such a picture is justified. We find the LLL to be separated from the rest by a spectral gap in the two-dimensional Dirac operator and analyze the corresponding LLL signature in four dimensions. We determine to what extent the quark condensate is LLL dominated at strong magnetic fields.
\end{abstract}

\section{QCD with strong magnetic fields}

The interplay of strong interactions with strong (electro-)magnetic fields plays an important role in physical systems such as the early universe, magnetized neutron stars and heavy-ion collisions. An effect discussed early on is the enhancement of the quark condensate by external magnetic fields coined magnetic catalysis $[1,2]$. It holds in many effective approaches to QCD at zero temperature. In this context, QCD in thermal equilibrium with external magnetic fields $B$ that are constant in space and time is probably the cleanest theoretical situation. First, it is amenable to lattice QCD simulations ${ }^{1}$, and, therefore, our understanding of QCD can be tested in this regime (even quantitatively). Besides confirming magnetic catalysis at low temperature, lattice simulations have revealed a surprise at temperatures $T$ around the QCD transition: inverse magnetic catalysis, i.e., a decrease of the condensate with increasing magnetic field $[3,4]$. As a result, the quark condensate has a nontrivial dependence on $T$ and $B$, and the pseudo-critical temperature of the QCD crossover decreases with the magnetic field [3], see Fig. 1. These effects do not occur for simulations on coarse lattices and higher-than-physical quark masses [5] and were missed in various non-lattice approaches.

\footnotetext{
a e-mail: falk.bruckmann@ur.de;speaker

${ }^{1}$ In contrast to a baryon chemical potential, a constant (electro-)magnetic field does not induce a complex action/sign problem.
} 

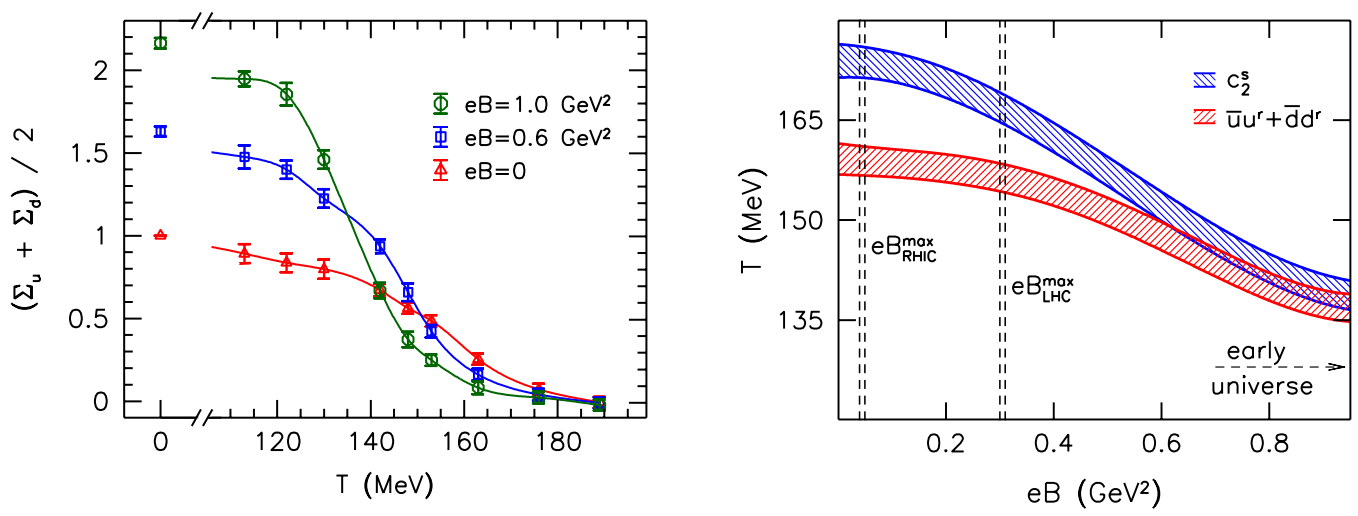

Figure 1. Left: the renormalized light quark condensate as a function of the temperature for three values of the magnetic field (data from [4]). It reveals magnetic catalysis at low temperatures and inverse magnetic catalysis around the transition. With increasing $B$ the inflection point moves to lower temperatures, which is reflected on the right panel: the pseudo-critical temperature - together with that obtained from the strange quark susceptibility, both in the continuum limit - decreases with the magnetic field [3].

\section{The Landau level picture}

In contrast to the strongly coupled case discussed so far, let us now come to the 'opposite' picture when magnetic fields act on otherwise free particles. Let $q$ denote the particle's electric charge and let the magnetic field point in the $z$-direction, $\vec{B}=(0,0, B)$. When restricting to the $(x, y)$-plane, classical particles move on a circle. Quantum mechanically this motion is quantized and includes a zero point energy plus a Zeeman effect acting on the spin $s_{z}$ along the magnetic field. For massless fermions in two dimensions the eigenvalues of (minus) the Dirac operator (squared) are

$$
-\not D^{2} \rightarrow \lambda^{2}=|q B| \cdot \underbrace{\left(2 n+1-2 \operatorname{sign}(q B) s_{z}\right)}_{2 k=0,2, \ldots}, \quad n=0,1, \ldots, \quad s_{z}= \pm 1 / 2 .
$$

The index $k$ denotes the Landau levels [6]. The lowest Landau level (LLL) with $k=0$ has zero eigenvalue ( $n=0$ and $2 s_{z}=\operatorname{sign}(q B)$, i.e., spin along $B$ for positive charge).

Moreover, these eigenvalues have a degeneracy proportional to $B$. To be precise, the magnetic field in a finite area of extent $L \times L$ with periodic boundary conditions is quantized ${ }^{2}$ in such a way that the magnetic flux is basically an integer [7]:

$$
\Phi=q B L^{2}=2 \pi N_{\Phi}, \quad N_{\Phi} \in \mathbb{Z} .
$$

For higher Landau levels one has to take into account that a fixed $k$ is obtained for two pairs of $\left(n, s_{z}\right)$, and the degeneracy of the Landau levels reads

$$
v_{k}=N_{\Phi} \cdot \begin{cases}1 & \text { for } k=0 \\ 2 & \text { for } k>0\end{cases}
$$

In QCD, a color factor $N_{c}=3$ has to multiply $v_{k}$ as well.

\footnotetext{
${ }^{2}$ like momentum is quantized on an interval
} 
Actually, the degeneracy $v_{0}=N_{\Phi}$ reflects the index theorem in two dimensions. On the one hand, the two-dimensional topological charge is the spatial integral over the field strength $B$ divided by $2 \pi$, i.e., just $N_{\Phi}$. According to the index theorem, this integer equals the difference of the numbers of fermionic zero modes of opposite chirality, in our case just the number of LLL modes having a definite spin polarization $s_{z}$ (the opposite $s_{z}$ does not lead to a zero mode).

\subsection{Obstructions against its applicability to QCD ...}

The Landau level picture introduced above applies to free fermions in two dimensions and in the continuum. Using it for realistic (lattice) QCD raises many questions about its applicability:

(a) When discretized on a lattice, not only the magnetic field becomes bounded from above $\mathrm{e}^{3}$, but the fermion spectrum becomes fractal (see below).

(b) For free fermions in four dimensions one has to add to $\lambda^{2}$ in Eq. (1) the contribution $p_{z}^{2}+p_{t}^{2}$ from the momenta corresponding to the extra directions. This inevitably mixes the Landau levels in the sense that each two-dimensional Landau level is scattered all over the ordered four-dimensional eigenvalues.

(c) Perhaps most importantly, QCD is a strongly coupled theory with the gluonic coupling among the quarks competing with the magnetic field effects. So the whole Landau level picture might be too simplistic.

When analyzing signatures of Landau levels in QCD thermodynamics, we will have to address these issues.

\section{$2.2 \ldots$ and evidence in favor of it}

Within the Landau level picture an attractive explanation of magnetic catalysis is the high degeneracy of Landau levels in the presence of magnetic fields. This applies in particular to the LLL, which, even if the eigenvalues are not exactly zero due to some perturbation, leads to an enhanced density of low modes and via the Banks-Casher relation [8] to an enhanced condensate. This mechanism could be at work in QCD, at least qualitatively. Actually, many non-lattice approaches rely on Landau levels or just the LLL, which is another motivation of this study.

On the lattice, evidence for Landau levels has been obtained from several quantities. First, the mass of the charged pion follows a LLL-like behavior. For such a scalar particle one has to consider the eigenvalues of the massive Laplacian, which are given by Eq. (1) setting $s_{z}=0$ and adding $m^{2}$ (and $p_{z}^{2}+p_{t}^{2}$, too). It means that the lowest level has eigenvalue $|q B|+m^{2}$ and indeed the pion mass follows $\sqrt{|e B|+m^{2}}$ [3]. That this relation holds even quantitatively is rooted in the weak coupling of the pions.

Secondly, the $B$-dependence of the Polyakov loop [9] and the field strength anisotropies [10] agrees qualitatively with the free case (worked out in appendices of these papers). As an example, the (squared and traced) color-electric field component parallel to $B$ has the smallest expectation values in lattice simulations, and in a one-loop calculation à la Euler-Heisenberg [11] with constant color fields this component has the largest effective action, i.e., is indeed the physically 'most expensive' component.

\footnotetext{
${ }^{3}$ The flux quantum number takes values from 0 to $N_{s}^{2}=(L / a)^{2}$. Again, such a bound is similar to that for momentum on a discretized space.
} 
Finally, the Landau level structure of the Dirac spectrum crucially influences the strong magnetic field behavior of the theory. In the asymptotic limit $B \rightarrow \infty$ the LLL-dominance becomes perfect and QCD turns into an anisotropic pure gauge theory [12]. This limiting theory can be directly simulated on the lattice and has a number of exciting features like the emergence of a first-order deconfinement phase transition [13].

\section{Outline and some technicalities}

In the remainder of this contribution we will identify the LLL in two-dimensional quark spectra, then transfer this feature to four dimensions and finally properly pose and answer the question, whether the LLL dominates the quark condensate.

Our analysis is based on lattice QCD simulations with 2+1 flavors of (rooted and smeared) staggered quarks at physical pion masses approaching the continuum limit, see [3] for more details. The quarks couple to the magnetic field with their electric charges $q_{u}=2|e| / 3$ and $q_{d}=q_{s}=-|e| / 3$. In particular, the weights and condensates of the $\mathrm{u}$ - and d-quark are different. All results shown below are for the d-quark (which also governs the flux quantization).

Eigenvalues and eigenmodes of the Dirac operator with magnetic field have been evaluated on configurations generated at zero magnetic field. This is the so-called valence quark effect. The full quark condensate is approximately the sum of the valence and the sea quark contribution [14]. For the latter the magnetic field is included just in the generation of the configurations through the quark determinant. This feedback on the system, as reflected in gluonic quantities such as the Polyakov loop, can be shown to be responsible for inverse magnetic catalysis [9]. We will restrict ourselves to the valence effect, since the identification of Landau levels is technically much simpler for that contribution.

\section{Spectra in two dimensions}

Fig. 2 shows eigenvalues of free fermions as a function of the magnetic field (as discussed in Sec. 2 in the continuum) on a $16 \times 16$ lattice. It reveals a fractal structure first found by Hofstadter [15]; for the precise mapping of our relativistic fermions onto Hofstadter's butterfly see [16].

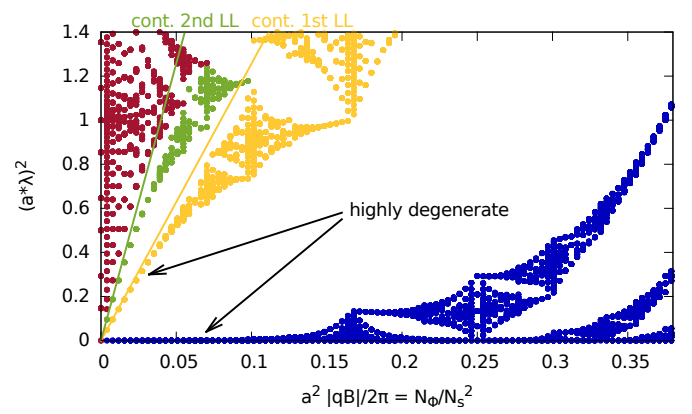

Figure 2. Spectra of free fermions as a function of the magnetic field on a $16 \times 16$ lattice, see text. The first $N_{\Phi}$ (agreeing with the LLL degeneracy $v_{0}$, see Eq. (3)) eigenvalues are plotted in blue, the next $2 N_{\Phi}=v_{1}$ eigenvalues in yellow etc. 
Both quantities in this figure are plotted in units of the inverse lattice spacing $a$ (which means that the values on the $x$-axis are quantized in units of $1 / 16^{2} \approx 0.004$ and bounded by 1 ). The continuum is reached in the lower left corner. In fact, the continuum relation of Eq. (1), i.e., zero eigenvalue for the LLL and $(a \lambda)^{2}=4 \pi \cdot a^{2}|q B| / 2 \pi$ for the first Landau level etc., fits the data well in this area. In other words, bunches of (sometimes highly degenerate) eigenvalues belonging to different continuum Landau levels are separated by gaps ${ }^{4}$. Moreover, the numbers of eigenvalues in these bunches agree with the continuum degeneracies $v_{k}$ of Eq. (3), as reflected in the color coding of Fig. 2.

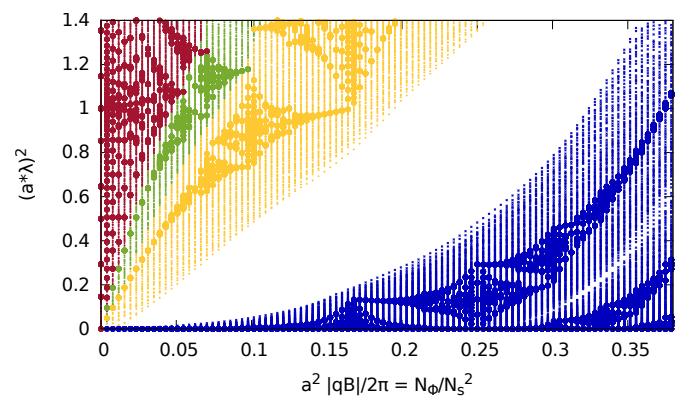

Figure 3. Quark spectra on a two-dimensional slice of a QCD configuration (thin dots) which can be viewed as having smeared out the analogous free spectrum of Fig. 2 (thick dots), but keeping a gap between the lowest modes/the LLL and the rest. As in Fig. 2 the color coding reflects the degeneracies of the Landau levels.

Next we switch on QCD interactions: we measure the eigenvalues on a $(x, y)$-plane of a typical QCD configuration, i.e., with SU(3) links in addition to the phase factors that implement the magnetic fields [17]. Taking fermion doublers, color factors and the fractional electric charge of the quark into account, Fig. 3 shows the resulting spectra. The QCD 'perturbation' has smeared out many structures of the fractal up to one important feature: there remains a sizeable gap between low eigenvalues and the rest. Furthermore, the number of these low eigenvalues again agrees with the continuum degeneracy $N_{\Phi}=v_{0}$ of the LLL. Therefore, the LLL can be identified in realistic QCD configuration, when restricting the latter to $(x, y)$-planes.

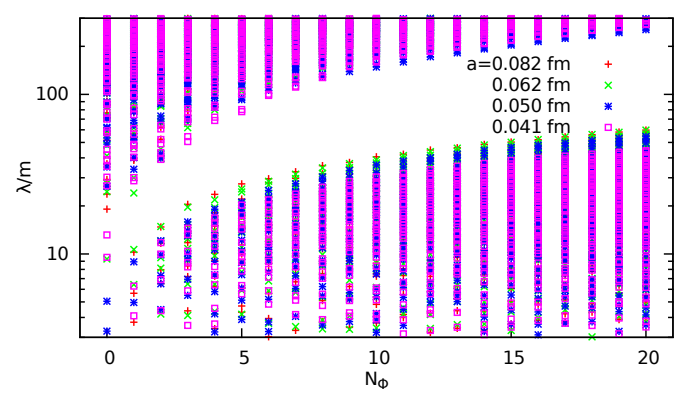

Figure 4. The gap in the two-dimensional quark spectra (see Fig. 3) for different lattice discretizations towards the continuum at fixed physical volume, where $N_{\Phi} \sim \Phi \sim B$.

\footnotetext{
${ }^{4}$ Since the graph is fractal, smaller gaps also appear within Landau levels. We view these as discretization artefacts.
} 
We conjecture that this gap is connected to the index theorem topologically protecting exactly $N_{\Phi}$ zero modes in the continuum and being insensitive to higher Landau levels. Note that the twodimensional index theorem is not modified by $\mathrm{SU}(3)$ field strengths due to their vanishing trace.

As a final check we show the behavior of this gap in the continuum limit. To this end we increase the lattice extensions $N_{t}, N_{s}$ and the lattice coupling $\beta$ (and adjust the lattice quark masses $\operatorname{am}(\beta)$ accordingly) keeping the physical volume and temperature constant. As a function of the magnetic field - or equivalently the flux $\Phi$ - we plot in Fig. 4 the lowest lattice eigenvalues in units of the bare light quark mass, $a \lambda / a m=\lambda / m$, which renders the corresponding density and spectral gap renormalization-group invariant $[18,19]$. As the figure shows, the gap survives the continuum limit and, therefore, is physical.

\section{Intermezzo: Localization}

The spectrum of the lattice Dirac operator can be investigated by means of Random Matrix Theory. Particularly interesting are spectral gaps near which the spectral statistics of the eigenvalues and the localization properties of the eigenmodes generically change. A measure for the latter is the inverse participation ratio (IPR),

$$
\operatorname{IPR}[\phi]=\sum_{i=1}^{N}|\phi(i)|^{4} \quad \text { assuming } \sum_{i=1}^{N}|\phi(i)|^{2}=1,
$$

where $i$ denotes the sites and $N$ their total number. The IPR is large and volume-independent for localized modes and small and vanishing as the volume increases for extended wave-like modes ${ }^{5}$.

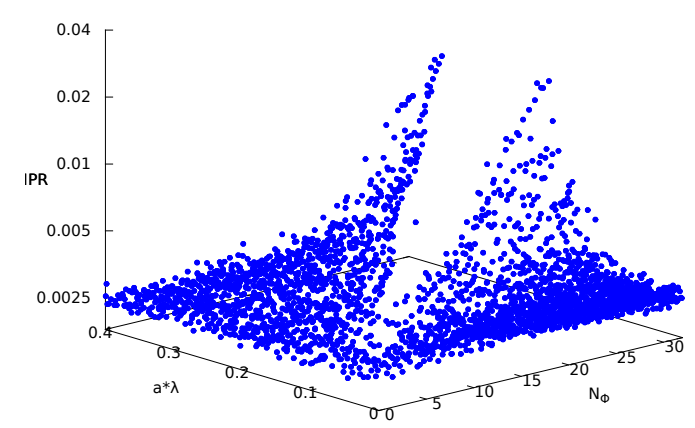

Figure 5. IPR (on a logarithmic scale) of two-dimensional quark modes as a function of magnetic field and eigenvalue.

Our findings for the IPRs of the two-dimensional quark modes are shown in Fig. 5. Clearly, the eigenmodes near the gap have a larger IPR, which hints at localization. To show that this is indeed the case, the scaling of the IPR with the volume of the system should be investigated. Localization is known to occur in the famous Anderson model [20] at the edge of the conduction band and in high temperature QCD [19, 21], near the (possibly soft) gap indicating the restoration of chiral symmetry. The presence of a mobility edge between localized and extended modes signals a second order phase transition in the spectrum [22]. Localization near the edge of the LLL caused by magnetic fields could be a peculiar feature, since the presence of Anderson localization in a two-dimensional system is not common [23]. This issue certainly deserves further studies.

\footnotetext{
${ }^{5}$ The maximal IPR $=1$ is reached for $\phi=1$ at one site and zero elsewhere, the minimal IPR $=\frac{1}{N}$ is taken for constant $|\phi|$.
} 


\section{Transfer to four dimensions}

We have already argued for the free case in Sec. 2.1 that Landau levels get mixed in the fourdimensional spectra. Therefore, it is not surprising that gaps in the four-dimensional quark spectra were found only for huge magnetic fields.

An alternative way to see Landau level signatures is based on a decomposition of the Dirac operator into the two directions $(x, y)$ of the external field strength $F_{x y}=B$ and the remainder. In the continuum this means:

$$
\not D=\sum_{\mu=x, y} \gamma^{\mu} D_{\mu}+\sum_{\mu=z, t} \gamma^{\mu} D_{\mu}=\not D_{x y}+\text { remainder },
$$

where $I_{x y}$ contains only links $U_{\mu}(x, y, z, t)$ with direction index $\mu \in\{x, y\}$ and thus is block-diagonal with respect to two-dimensional slices of fixed $(z, t)$,

$$
\not D_{x y}=\oplus_{z, t} D_{x y}(z, t) \text {. }
$$

Only the links with $\mu \in\{z, t\}$ in the remaining part connect different slices. A completely analogous decomposition exists for the staggered Dirac operator that we use ${ }^{6}$.

In Sec. 4 we have collected evidence for the LLL in the corresponding two-dimensional eigenmodes $\phi_{j}$,

$$
\not D_{x y}(z, t) \phi_{j}(z, t)=i \hat{\lambda}_{j}(z, t) \phi_{j}(z, t) .
$$

From the construction it is clear that the modes $\phi_{j}(z, t)$ for all indices $j$ and on all slices $(z, t)$ form a complete four-dimensional basis. Therefore, it is reasonable to analyze the projection of eigenmodes $\psi$ of the full Dirac operator $\not D$ onto subsets of $\phi_{j}$ 's, which involves overlaps

$$
W_{j}=\sum_{z, t}\left|\left\langle\phi_{j}(z, t) \mid \psi\right\rangle\right|^{2}
$$

with the normalization $\sum_{j} W_{j}=1$. Note that both kind of eigenmodes change with the magnetic field. We have summed over the slices $(z, t)$, which are physically equivalent, and expect signatures of the LLL especially in the dependence on the index $j$. For a reduction of statistical errors, we average $W_{j}$ over $\psi$ 's with similar eigenvalues.

\footnotetext{
${ }^{6} \mathrm{~A}(x, y)$-part can also be defined for the Wilson-Dirac operator.
}

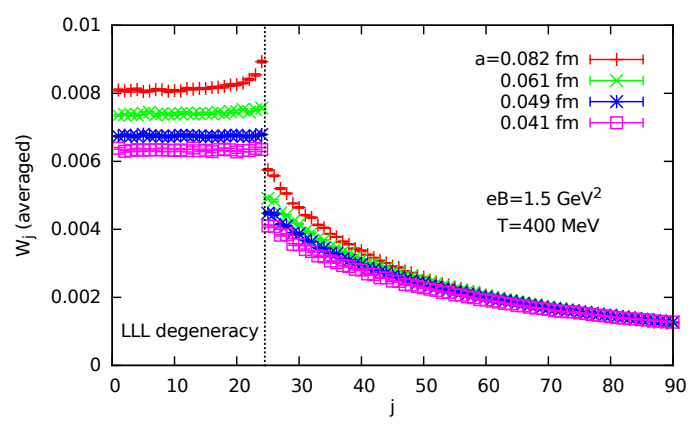

Figure 6. Averaged overlaps $W_{j}$ of four-dimensional modes $\psi$ with eigenvalues in the low range $\lambda / m \in[220,225]$ and the two-dimensional modes $\phi_{j}$, as a function of the index $j$, see Eqs. (5-8). In the two-dimensional modes the LLL can be identified and the end of the corresponding $j$-range, $N_{c} N_{\Phi}=24$, is marked by a vertical line. 
Fig. 6 shows these overlaps for low four-dimensional modes. The overlap with the LLL modes is almost constant, whereas the overlap with higher two-dimensional modes is significantly smaller. The latter might have been expected, but the end of the LLL is clearly signalled by a jump in the overlap, which remains towards the continuum. (We expect all these features to disappear when the magnetic field is diminished.) Hence, we have found a way to identify LLL signatures in four dimensions, which allows us to pose the question of its dominance.

\section{LLL projection in the condensate and its potential dominance}

These findings motivate the definition of a LLL projection for all quantities that have a spectral decomposition, i.e., that are functions of the Dirac operator. The quark condensate reads

$$
\langle\bar{\Psi} \Psi\rangle=\frac{T}{V}\left\langle\operatorname{Tr} \frac{1}{\not D+m}\right\rangle=\frac{T}{V}\left\langle\operatorname{Tr} \frac{m}{-\not D^{2}+m^{2}}\right\rangle=\frac{T}{V}\left\langle\sum_{\lambda>0} \frac{2 m}{\lambda^{2}+m^{2}}\right\rangle,
$$

where we have used the chiral symmetry of $\not D$. In the staggered formalism the existence of partners with eigenvalue $\pm i \lambda$ is guaranteed due to the $\eta_{5}$-symmetry. Moreover, we have assumed that exact zero modes are absent, which is generic.

The LLL projection we define as

$$
\langle\bar{\Psi} \Psi\rangle_{L L L}=\frac{T}{V}\left\langle\operatorname{Tr} \frac{m}{-\not^{2}+m^{2}} P_{L L L}\right\rangle
$$

with the projector

$$
P_{L L L}=\sum_{j=1}^{N_{c} N_{\Phi}} \sum_{\text {doublers }} \sum_{z, t}\left|\phi_{j}(z, t)\right\rangle\left\langle\phi_{j}(z, t)\right|,
$$

where the sum also includes the two-dimensional doublers. This projection can be generalized to quark bilinears $\langle\bar{\Psi} \Gamma \Psi\rangle$. The projected condensate

$$
\langle\bar{\Psi} \Psi\rangle_{L L L}=\frac{T}{V}\langle\sum_{\lambda>0} \frac{2 m}{\lambda^{2}+m^{2}} \sum_{j=1}^{N_{c} N_{\Phi}} \sum_{\text {doublers }} \overbrace{\sum_{z, t}\left|\left\langle\phi_{j}(z, t) \mid \psi_{\lambda}\right\rangle\right|^{2}}^{W_{j}}\rangle
$$

contains the overlaps $W_{j}$ from the previous section ${ }^{7}$, which are particularly large for the $j$-range involved here.

Since both the full and the projected condensate are divergent in the continuum limit, what remains to be discussed is their renormalization. For the full condensate the additive divergence is usually removed by subtracting the $B=0$-case defining the following difference:

$$
\Delta\langle\bar{\Psi} \Psi\rangle=\langle\bar{\Psi} \Psi\rangle(B)-\langle\bar{\Psi} \Psi\rangle(0) .
$$

Since the magnetic field has only a small effect on the high modes, this contribution, which is responsible for the additive divergence, is removed. Let us stress one subtlety here: the notion of Landau levels is meaningless at $B=0$ and nothing would be subtracted from $\langle\bar{\Psi} \Psi\rangle_{L L L}$, since the $j$-range vanishes. Instead, we subtract from $\langle\bar{\Psi} \Psi\rangle_{L L L}(B)$ a condensate of the form (12) with the index range given

\footnotetext{
${ }^{7}$ In Eq. (12) we have written $\psi_{\lambda}$ in order to stress the connection to the eigenvalues $\lambda$.
} 


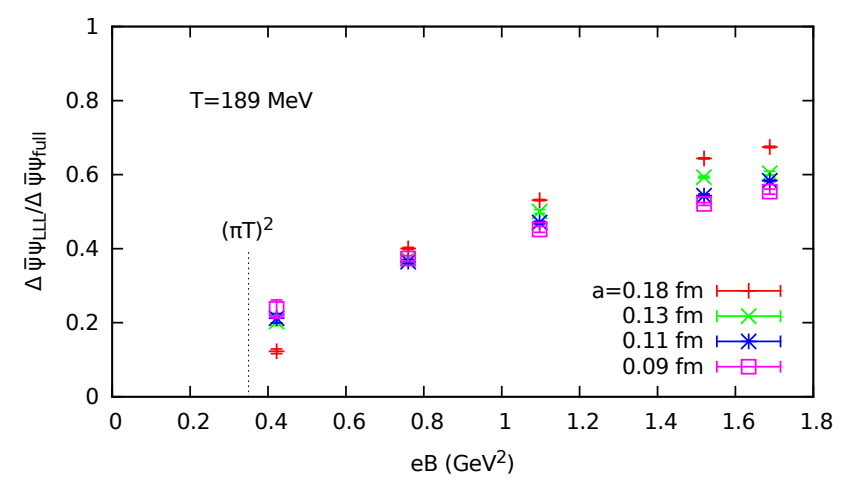

Figure 7. The ratio of LLL projected vs. full condensate defined in Eq. (14), at a fixed temperature slightly above the QCD transition, as a function of the magnetic field. The lattice spacings are decreased keeping the physical volume fixed. The Matsubara frequency $\pi T$, squared to match the dimension of $e B$, is marked as well (the squares of $\Lambda_{Q C D}$ and of the pion mass are considerably smaller).

by the nonzero magnetic field (its flux quantum number $N_{\Phi}$ ), while the other quantites $\lambda, \phi_{j}(z, t)$ and $\psi_{\lambda}$ are computed at $B=0$. An alternative additive renormalization is to use the gradient flow [24].

The multiplicative divergence can be cured in both cases by multiplying with the bare mass, and so it also disappears in the ratio

$$
\frac{\Delta\langle\bar{\Psi} \Psi\rangle_{L L L}}{\Delta\langle\bar{\Psi} \Psi\rangle}
$$

which is our final observable to determine a possible LLL dominance of the condensate.

Fig. 7 shows the results for this ratio at a fixed temperature as a function of the magnetic field. The ratio is roughly $1 / 2$ for the values of $B$ shown here ${ }^{8}$. These $B$-values exceed the Matsubara frequency (squared), such that the magnetic field is the largest energy scale in the system. The increase of the ratio with $B$ is rather slow and it is not even obvious that for huge values of $B$ the ratio becomes 1 indicating full dominance/saturation. To understand this, we have computed the same ratio for the free case and found $1-O\left(1 / \log \left(|q B| / m^{2}\right)\right)$ as the large- $B$ behavior at zero temperature, which also approaches full dominance very slowly. We have also computed numerically the same ratio in two dimensions (in the $(x, y)$-plane), where it is always very close to 1.

The picture outlined here does not depend much on the temperature. For more results and details, including the spin projection $\left\langle\bar{\Psi} \sigma_{x y} \Psi\right\rangle$, we refer to a forthcoming publication.

\section{Summary and outlook}

We have studied quark spectra in equilibrium QCD with constant external magnetic fields.

Of the Landau levels from the free two-dimensional case, (only) the LLL can be identified by a spectral gap right after the correct amount of eigenmodes corresponding to the LLL degeneracy, which is proportional to the magnetic flux. We have argued that this mechanism is due to the twodimensional index theorem and showed that modes near the gap are more localized.

Our identification of the LLL in four-dimensional spectra is based on a decomposition of the Dirac operator into the $(x, y)$-directions, i.e., acting on two-dimensional slices of fixed $(z, t)$, and the

\footnotetext{
${ }^{8}$ These $B$-values display the effects of inverse magnetic catalysis and decreasing $T_{c}$ in Fig. 1 .
} 
remainder. The overlap between the four-dimensional eigenmodes and those of the two-dimensional Dirac operator reveals a jump at the LLL degeneracy.

With the corresponding range of these slice-eigenmodes we have defined the LLL projection of quark observables. In the - properly renormalized - condensate the LLL gives a non-negligible contribution when the magnetic field is the largest scale, but does not dominate the condensate either. The temperature dependence of this effect seems mild. We hope that our findings serve as benchmarks or input for non-lattice approaches.

A technically more involved question is what role Landau levels play in the sea quark effects, with which one could determine, e.g., LLL dominance of inverse magnetic catalysis.

\section{Acknowledgements}

We acknowledge support by DFG (BR 2872/6-1, EN 1064/2-1, SFB/TRR 55 and BR 2872/7-1), by OTKA (OTKA-K-113034) and by the Hungarian Academy of Sciences ( "Lendület" LP2011-011).

\section{References}

[1] S. Schramm, B. Müller, A.J. Schramm, Mod. Phys. Lett. A 7, 973 (1992).

[2] V.P. Gusynin, V.A. Miransky, I. A. Shovkovy, Nucl. Phys. B 462, 249 (1996) [hep-ph/9509320].

[3] G.S. Bali, F. Bruckmann, G. Endrődi, Z. Fodor, S.D. Katz, S. Krieg, A. Schäfer, K.K. Szabó, JHEP 1202, 044 (2012) [1111.4956].

[4] G.S. Bali, F. Bruckmann, G. Endrődi, Z. Fodor, S.D. Katz, A. Schäfer, Phys. Rev. D 86, 071502 (2012) [1206.4205].

[5] M. D’Elia, S. Mukherjee, F. Sanfilippo, Phys. Rev. D 82, 051501 (2010) [1005.5365].

[6] L. Landau, Zeitschrift für Physik A, Hadrons and Nuclei 64, 629 (1930).

[7] G. 't Hooft, Nucl. Phys. B 153, 141 (1979).

[8] T. Banks, A. Casher, Nucl. Phys. B 169, 103 (1980).

[9] F. Bruckmann, G. Endrődi, T.G. Kovács, JHEP 1304, 112 (2013) [1303.3972].

[10] G.S. Bali, F. Bruckmann, G. Endrődi, F. Gruber, A. Schäfer, JHEP 1304, 130 (2013) [1303.1328].

[11] W. Heisenberg, H. Euler, Z. Phys. 98, 714 (1936) [physics/0605038].

[12] V.A. Miransky, I.A. Shovkovy, Phys. Rev. D 66, 045006 (2002) [hep-ph/0205348].

[13] G. Endrődi, JHEP 1507, 173 (2015) [1504.08280].

[14] M. D’Elia, F. Negro, Phys. Rev. D 83, 114028 (2011) [1103.2080].

[15] D.R. Hofstadter, Phys. Rev. B 14, 2239 (1976).

[16] G. Endrődi, PoS LATTICE 2014, 018 (2014) [1410.8028].

[17] M.H. Al-Hashimi, U.-J. Wiese, Annals Phys. 324, 343 (2009) [0807.0630].

[18] L. Giusti, M. Lüscher, JHEP 0903, 013 (2009) [0812.3638].

[19] T.G. Kovács, F. Pittler, Phys. Rev. D 86, 114515 (2012) [1208.3475].

[20] P.W. Anderson, Phys. Rev. 109, 1492 (1958).

[21] A.M. García-García, J.C. Osborn, Phys. Rev. D 75, 034503 (2007) [hep-lat/0611019].

[22] M. Giordano, T.G. Kovács, F. Pittler, Phys. Rev. Lett. 112, 102002 (2014) [1312.1179].

[23] B. Kramer, A. MacKinnon, T. Ohtsuki, K. Slevin, Int. J. Mod. Phys. 24, 1841 (2010) [1004.0285].

[24] M. Lüscher, JHEP 1008, 071 (2010), erratum: JHEP 1403, 092 (2014) [1006.4518]. 